\title{
Evaluating the Prevalence of Foodborne Pathogens in Livestock Using Metagenomics Approach
}

\author{
Hyeri Kim ${ }^{1 \dagger}$, Jin Ho Cho ${ }^{2 \dagger}$, Minho Song ${ }^{3 \dagger}$, Jae Hyoung Cho ${ }^{1}$, Sheena Kim ${ }^{1}$, Eun Sol Kim ${ }^{1}$, \\ Gi Beom Keum ${ }^{1}$, Hyeun Bum Kim ${ }^{1 *}$, and Ju-Hoon Lee ${ }^{4 *}$ \\ ${ }^{1}$ Department of Animal Resources Science, Dankook University, Cheonan 31116, Republic of Korea \\ ${ }^{2}$ Division of Food and Animal Science, Chungbuk National University, Cheongju 28644, Republic of Korea \\ ${ }^{3}$ Division of Animal and Dairy Science, Chungnam National University, Daejeon 34134, Republic of Korea \\ ${ }^{4}$ Department of Food Animal Biotechnology, Department of Agricultural Biotechnology, Center for Food and \\ Bioconvergence, Seoul National University, Seoul 08826, Republic of Korea
}

\begin{abstract}
Food safety is the most important global health issue due to foodborne pathogens after consumption of contaminated food. Foodborne bacteria such as Escherichia coli, Salmonella enterica, Staphylococcus aureus, Campylobacter spp., Bacillus cereus, Vibrio spp., Yersinia enterocolitica and Clostridium perfringens are leading causes of the majority of foodborne illnesses and deaths. These foodborne pathogens often come from the livestock feces, thus, we analyzed fecal microbial communities of three different livestock species to investigate the prevalence of foodborne pathogens in livestock feces using metagenomics analysis. Our data showed that alpha diversities of microbial communities were different according to livestock species. The microbial diversity of cattle feces was higher than that of chicken or pig feces. Moreover, microbial communities were significantly different among these three livestock species (cattle, chicken, and pig). At the genus level, Staphylococcus and Clostridium were found in all livestock feces, with chicken feces having higher relative abundances of Staphylococcus and Clostridium than cattle and pig feces. Genera Bacillus, Campylobacter, and Vibrio were detected in cattle feces. Chicken samples contained Bacillus, Listeria, and Salmonella with low relative abundance. Other genera such as Corynebacterium, Streptococcus, Neisseria, Helicobacter, Enterobacter, Klebsiella, and Pseudomonas known to be opportunistic pathogens were also detected in cattle, chicken, and pig feces. Results of this study might be useful for controlling the spread of foodborne pathogens in farm environments known to provide natural sources of these microorganisms.
\end{abstract}

Received: September 17, 202 Accepted: October 13, 2021

First published online: October 15, 2021

${ }^{*}$ Corresponding authors H.B. Kim

Phone: +82-41-550-3653

E-mail: hbkim@dankook.ac.kr J.H. Lee

Phone: $+82-2-880-4854$

E-mail: juhlee@snu.ac.kr

${ }^{\dagger}$ These authors contributed equally to this work.

Supplementary data for this paper are available on-line only at http://jmb.or.kr.

pISSN 1017-7825 elSSN 1738-8872

Copyright $\odot 2021$ by the authors. Licensee KMB. This article is an open access article distributed under the terms and conditions of the Creative Commons Attribution (CC BY) license.
Keywords: Foodborne-pathogens, food safety, livestock, metagenomics, 16S rRNA gene

\section{Introduction}

Foodborne illness represents a major threat to public health and is frequently attributed to pathogenic microorganisms in livestock feces. Leading causes of the majority of foodborne illnesses and deaths include foodborne pathogens, such as Escherichia coli, Salmonella enterica, Staphylococcus aureus, Campylobacter spp., Bacillus cereus, Vibrio spp., Yersinia enterocolitica and Clostridium perfringens [1, 2]. In South Korea, Food and Drug Safety institute reported the number of foodborne illness cases in the past seven years (2012-2019). Although the number of outbreaks has fluctuated from year to year, there has been a general increasement. Especially, there was an increase of $29.3 \%$ in the number of outbreaks (697 cases) in 2018 compared to that in the previous year (539 cases) in South Korea.

Foodborne illness outbreaks could be attributed to insufficient heating of food and cross-contamination during the food supply process. Especially, contamination of foodborne pathogens can occur from a farm environment, such as livestock feces throughout the processing of abattoirs and packaging to markets or restaurants. Foodborne pathogens originated from livestock feces can grow in a contaminated food at any point along the food supply process [3-5]. Therefore, the understanding the public health risk of microorganisms in livestock feces is essential process for the prevention of foodborne illness. The initial step to access the public health risk of microorganisms in livestock feces is to identify and describe microbial communities including foodborne pathogens. Conventional methods to identify bacterial pathogens from various sources depend on culturing on agar plates. However, these method takes 2-3 days to culture pathogens and up to more than a week for the confirmation of specific pathogenic microorganisms [6]. Currently, metagenomics sequencing techniques as culture-independent 
methods are widely used to identify and characterize microbiota [7]. Therefore, we hypothesized that the metagenomics approach could be used to detect foodborne pathogens in samples such as foods, feces, and soils $[8,9]$.

The purpose of this study was to investigate the prevalence of foodborne pathogens in the livestock feces using the metagenomics approach.

\section{Materials and Methods \\ Sample Collection}

The feces $($ Cattle $=33$, chicken $=33$, Pig=34) from the livestock were collected in South Korea. Samples were stocked immediately into $-80^{\circ} \mathrm{C}$ deep freezer to maintain stability of microbiota from the feces (Table S1).

\section{DNA Extraction}

Total DNA representing microbial community were extracted from $200 \mathrm{mg}$ of each feces sample by using QIAamp FAST DNA Stool Mini kit (Qiagen, Germany) according to the manufacturer's instructions with some modifications. Briefly, cell lysis was conducted with bead-beating the samples twice for $2 \mathrm{~min}$ at $300 \mathrm{rpm}$ instead of vortex during the DNA extraction process. The isolated DNA were quantified using a Colibri Microvolume Spectrometer (Titertek Berthold, Germany) and processed further analysis.

\section{6s rRNA Gene Sequencing}

Fifty ng of the total DNA from each feces sample was used to amplified the V5 to V6 [10] hypervariable regions of the 16S rRNA genes using the universal primer sets 799F (5'-CMGGATTAGATACCCKGG-3') and 1114R (5'GGGTTGCGCTCGTTGC-3') [11]. The PCR products were purified using the Wizard SV Gel and PCR CleanUp System (Promega Corp., USA). The 16S rRNA gene amplicons were sequenced using the Illumina MiSeq platform at Macrogen Inc. (Korea) according to the manufacturer's instructions.

\section{Bioinformatics Analysis}

All the sequence data from the Illumina Miseq platform with length of less than 200 base pair were trimmed to minimize the effects of random sequencing errors. Chimeric sequences were identified and eliminated from the sequences using the UCHIME algorithm implemented in Quantitative Insights into Microbial Ecology (QIIME) [12]. The QIIME pipeline was used to conduct Operational taxonomic unit (OTU) picking, taxonomic assignment, diversity analysis, and visualization. OTU picking was performed using de novo operational taxonomic unit (OTU) clustering with an OTU definition at an identity cutoff of 97\% [13]. Taxonomic assignment and classification were performed using the Ribosomal Database Project (RDP) classifier implemented in QIIME [14].

Microbial alpha diversity including Chao1, observed OTUs, Shannon and Simpson indices were calculated using QIIME. Beta-diversity was measured using both weighted and unweighted UniFrac distance metrics using QIIME [15]. Principal coordinate analysis (PCoA) plots were generated based on the weighted and unweighted UniFrac distance metrics. Analysis of similarities (ANOSIM) was used to determine whether the microbial compositions between the three groups were significantly different using QIIME and was based on the weighted and unweighted UniFrac distance metrics. The abundance of taxa among the fecal samples was compared using STAMP software [16]. Samples were compared by ANOVA, followed by the Tukey-Kramer post hoc test $(p<$ $0.05)$.

\section{Results}

\section{Sequencing Data}

16S rRNA gene amplicons from cattle, chicken, and pig feces were sequenced using an Illumina MiSeq platform. As a result, a total of $12,764,596$ DNA sequence reads were generated from animal feces after qualityfiltering. The average number of sequences generated per feces was 118,250 for cattle, 122,438 for chicken, and 142,503 for pig (Table S2).

\section{Alpha Diversity}

OTUs were analyzed for each sample at $97 \%$ identity to compare bacterial diversity and species richness between animal feces samples. While observed OTUs, Chao, and Shannon indices indicate the different number of bacterial species present in each livestock species, similar Simpson diversity indices among the livestock species indicate the comparable population size of each of the bacterial species present in three livestock species because Simpson index considers species evenness more than species richness (Table 1).

Table 1. Average values of Alpha-diversity measurements for feces of livestock.

\begin{tabular}{lcccc}
\hline & Observed OTUs & Chao & Shannon & Simpson \\
\hline Cattle & 20442.76 & 82076.52 & 11.33 & 1 \\
Chicken & 4536.545 & 13068.23 & 5.984739 & 0.93 \\
Pig & 6903.412 & 23217.42 & 7.170497 & 0.95 \\
\hline
\end{tabular}


A

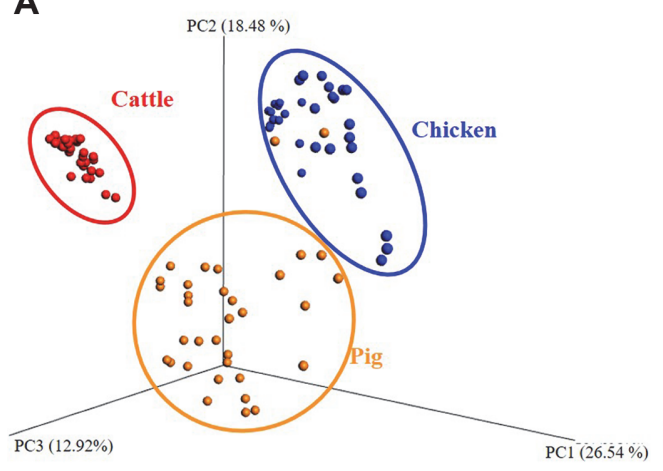

B

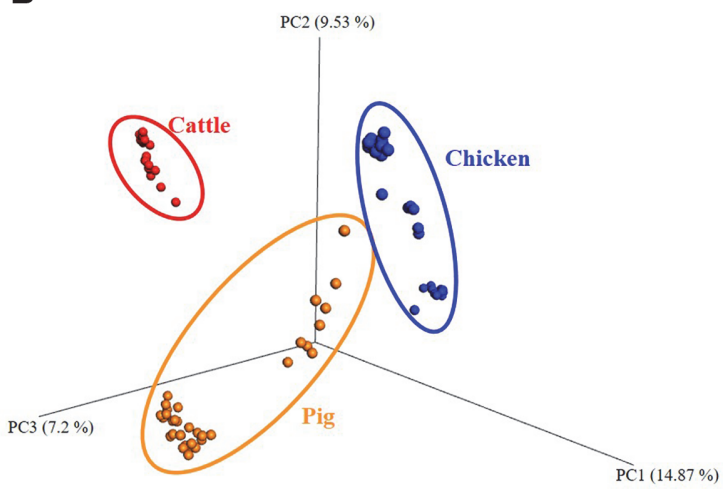

Fig. 1. Bacterial communities using Principal coordinate analysis (PCoA) plots based on (A) weighted and (B) unweighted UniFrac distance metrics. A three-dimensional PCoA plot of the Illumina sequencing data from animal feces $(n=100)$ was generated using the software package QIIME. Samples associated with cattle (clustered by the red ellipse), chicken (clustered by the blue ellipse), and pig (clustered by the yellow ellipse) are shown as single points.

\section{Beta Diversity}

Analysis of similarities using ANOSIM of unweighted UniFrac distances indicated that samples were significantly different $(p<0.05)$ with a relatively high R-value of 0.6936 , indicating that three species consisted of different microbiota. Weighted UniFrac distances from ANOSIM analysis also confirmed that distances of microbiota were similar to unweighted UniFrac distances, suggesting that microbial communities of the three species were significantly different $(p<0.05)$ with an R-value of 0.5181 . PCoA plot of unweighted UniFrac visually established separated distances among the three livestock species samples (Fig. 1).

\section{Relative Abundance}

A total of 37 phyla, 80 classes, 349 families, and 771 genera were identified in feces of these three different livestock species. Relative abundances of fecal microbiota of each livestock species at Phylum, Family, and Genus levels are shown in Fig. 2.

At the Phylum level, Firmicutes had the highest relative abundance in Cattle (73.87\%), followed by that in Chicken (64.80) and Pig (49.25\%) fecal microbiota. Bacteroidetes was the second abundant bacterial phylum (11.37\%) in cattle fecal microbiota. Phyla Proteobacteria (20.83\%), Bacteroidetes (7.64\%), Deferribacteres (2.25\%), and Actinobacteria (2.22\%) were detected in chicken feces. The pig fecal microbial community had phyla Bacteroidetes (21.21\%), Proteobacteria (19.75\%), and Spirochaetes (5.71\%) (Fig. 2A).

At the family level, cattle fecal microbiota mainly had Ruminococcaceae (58.86\%), Lachnospiraceae (10.19\%), Rikenellaceae (3.62\%), Spirochaetaceae (3.27\%), Bacteroidaceae (2.74\%), and Clostridiaceae (1.28\%). Chicken samples were predominantly composed of Lachnospiraceae (16.89\%), Lactobacillaceae (12.86\%), Ruminococcaceae (12.59\%), Enterobacteriaceae (9.65\%), Clostridiaceae (4.70\%), and Alcaligenaceae (2.08\%). Families Ruminococcaceae (20.79\%), Prevotellaceae (13.93\%), Lachnospiraceae (11.77\%), Succinivibrionaceae (10.98\%), Spirochaetaceae (6.19\%), Lactobacillaceae (4.70\%), Enterobacteriaceae (3.89\%), Bacteroidaceae (2.85\%), Clostridiaceae (2.59\%),

A

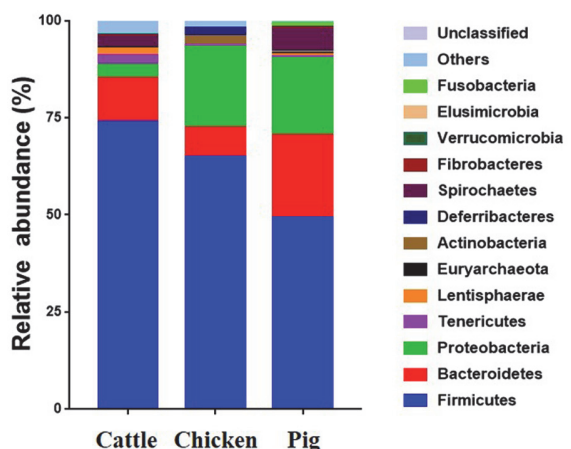

B

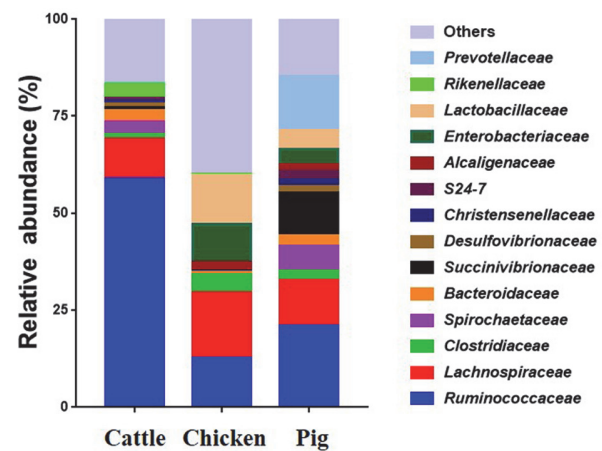

C

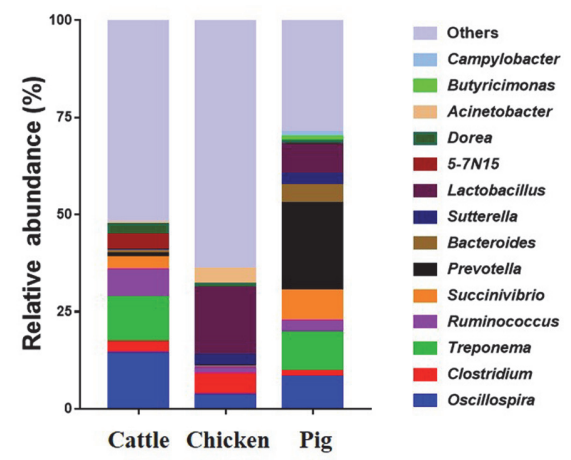

Fig. 2. Relative abundance of taxonomy at the (A) phylum, (B) family and (C) genus levels in feces of cattle, chicken and pig. Phylogenetic assignment and classification based on $16 \mathrm{~S}$ rRNA sequence similarity were conducted by using the Ribosomal Database Project (RDP) classifier implemented in QIIME. 


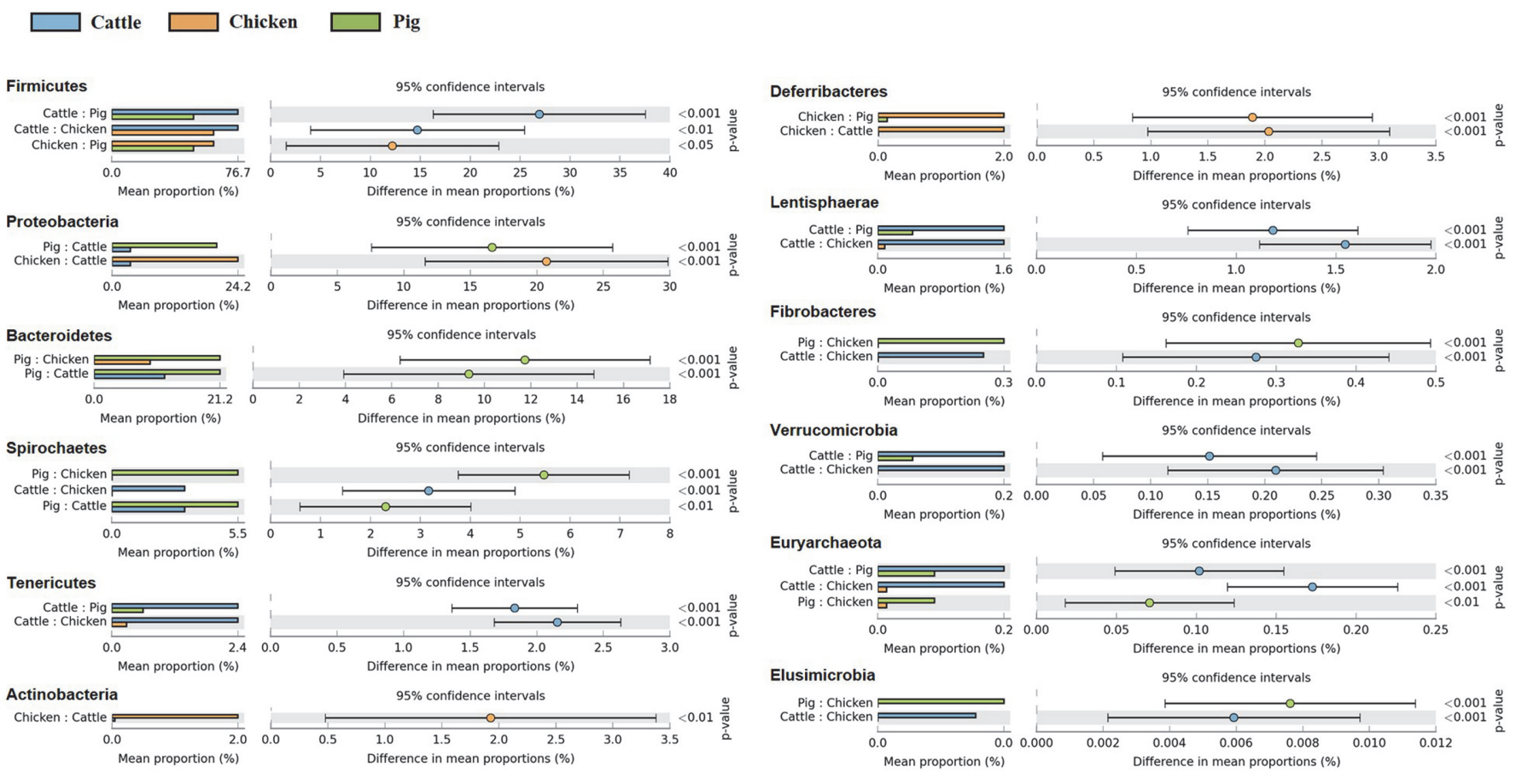

Fig. 3. Extended error plots identifying significantly different taxa at the phylum level. Samples were compared by ANOVA, followed by the TukeyKramer post hoc test without correction for multiple comparisons. Corrected $p$-Values are shown at the right.

and S24-7 (2.15\%) (Fig. 2B) were detected in pig fecal samples.

At the genus level, bacterial communities of different livestock species were composed of a variety of genera. Oscillospira and Treponema were the top two most significantly enriched genera in cattle fecal samples. Lactobacillus was the largest relative abundant genus in chicken fecal samples. Prevotella and Treponema were the most abundant genera in pig fecal samples (Fig. 2C).

Extended error bar plots were used to identify significantly different taxa at phylum, family, and genus levels among different fecal samples. At the phylum level, relative abundances of Firmicutes, Tenericutes, Lentisphaerae, Verrucomicrobia, and Euryarchaeota in cattle feces were significantly higher than those in the other two livestock species. In chicken feces, relative abundances of Proteobacteria, Actinobacteria, and Deferribacteres were statistically higher than those in other livestock species. In pig fecal samples, populations of Bacteroidetes and Spirochaetes were significantly larger than those in cattle and chicken fecal samples (Fig. 3). At the family level, relative abundances of Ruminococcaceae and Rikenellaceae were predominantly higher in cattle fecal samples than in chicken and pig fecal samples. Families Prevotellaceae, Succinivibrionaceae, Spirochaetaceae, S24-7, Christensenellaceae, and Desulfovibrionaceae were differentially abundant in pig feces than in other livestock species. However, Lactobacillaceae was the only family whose relative abundance was significantly higher in chicken feces than in the other two species (Fig. 4). At the genus level, Oscillospira, Treponema, Ruminococcus, 5$7 \mathrm{~N} 15$, and Dorea showed higher abundances in cattle feces than in chicken and pig feces. Relative abundances of Prevotella, Bacteroides, and Campylobacter were overwhelmingly higher in pig feces than in the other two species. Lactobacillus and Acinetobacter were more detected in chicken feces than in the other two (Fig. 5).

\section{Detection of Foodborne Pathogens in Three Different Livestock Feces}

In this study, we evaluated the prevalence of foodborne pathogens in livestock feces using the metagenomics approach. A total of 7 foodborne pathogens were detected in feces samples using 16S rRNA gene analysis. Staphylococcus and Clostridium were present in cattle, chicken, and pig feces. However, higher relative abundances of those genera were detected in chicken feces than in cattle and pig samples. In cattle feces, genera Bacillus, Campylobacter, and Vibrio were confirmed (Fig. 6A). Bacillus, Listeria, and Salmonella with low relative abundances were found in chicken feces (Fig. 6B). In pig feces, it was confirmed that Campylobacter had the highest relative abundance among detected foodborne pathogens. Salmonella was also detected (Fig. 3C). In addition, potential pathogens such as Corynebacterium, Streptococcus, Klebsiella, and Pseudomonas were confirmed in cattle, chicken, and pig feces (Figs. 3D, 3E, and 3F). Relative abundances of Streptococcus and Corynebacterium in chicken feces were higher than those in cattle and pig feces (Fig. 3E). Helicobacter was detected more in pig feces than in the other two (Fig. 3F). 

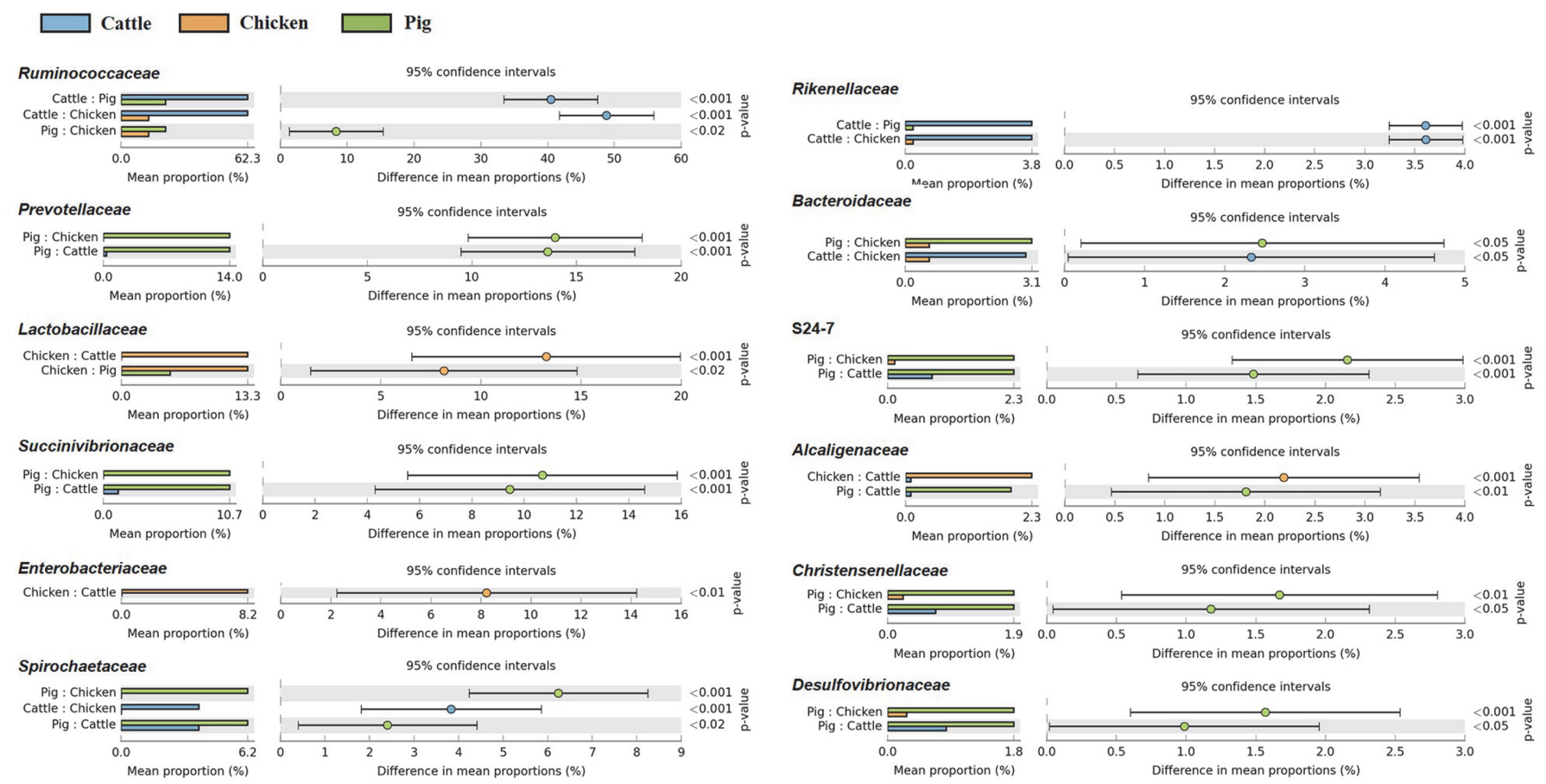

Fig. 4. Extended error plots identifying significantly different taxa at the family level. Samples were compared by ANOVA, followed by the TukeyKramer post hoc test without correction for multiple comparisons. Corrected $p$-Values are shown at the right.

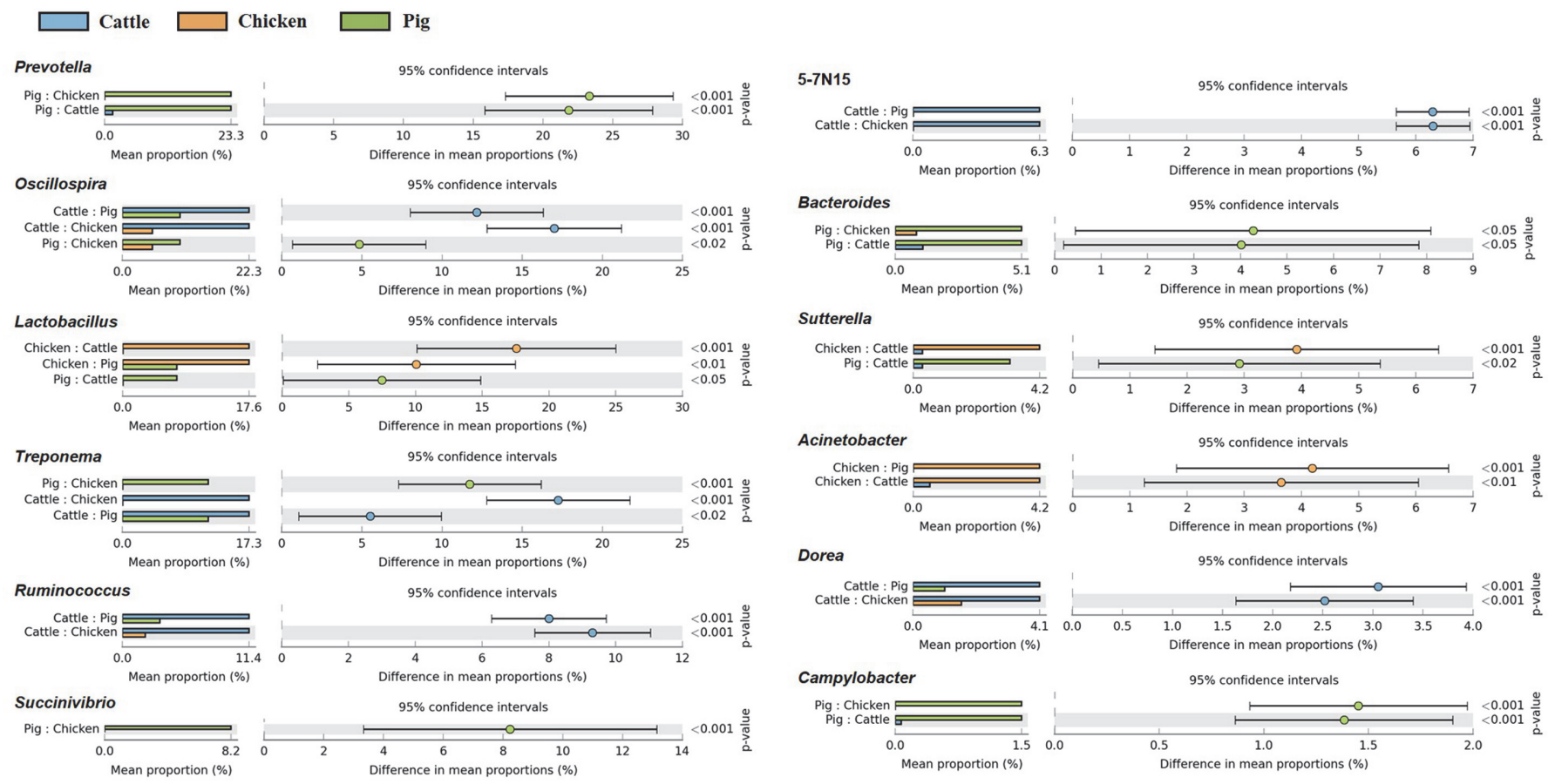

Fig. 5. Extended error plots identifying significantly different taxa at the genus level. Samples were compared by ANOVA, followed by the TukeyKramer post hoc test without correction for multiple comparisons. Corrected $p$-Values are shown at the right.

\section{Discussions}

This study was conducted to evaluate contamination levels of foodborne pathogens in feces of livestock animals such as cattle, chicken, and pig. It has been reported that some of pathogens, such as Salmonella and 
A

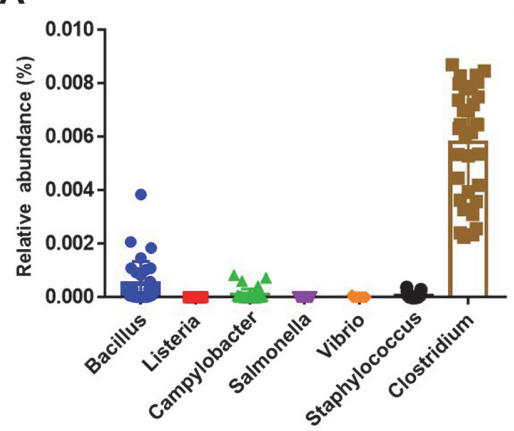

D

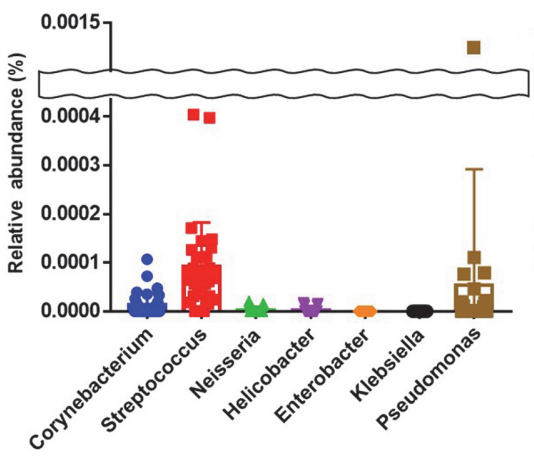

B
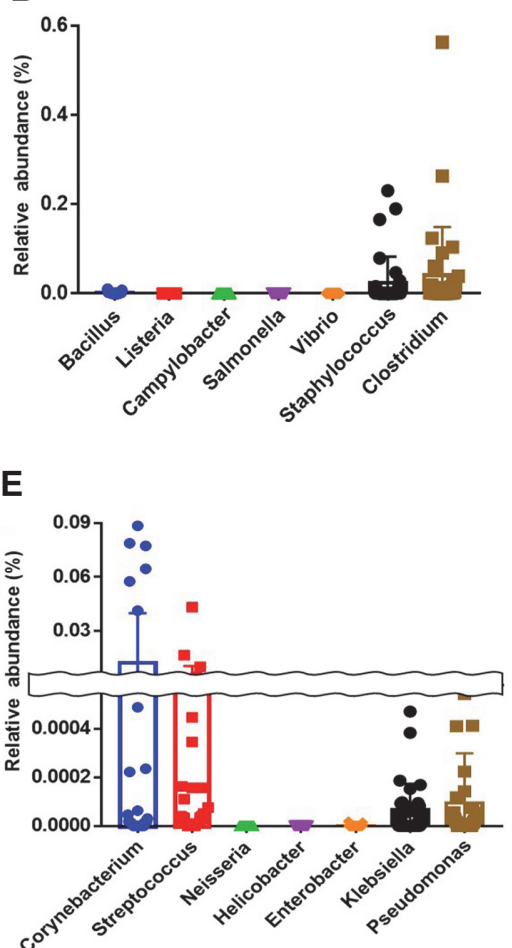

C

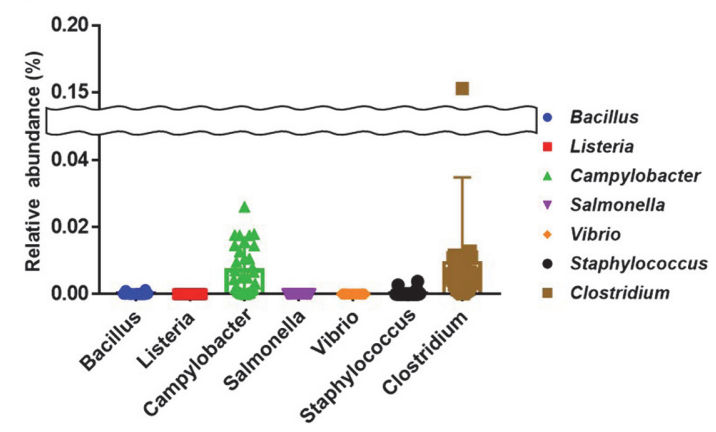

F

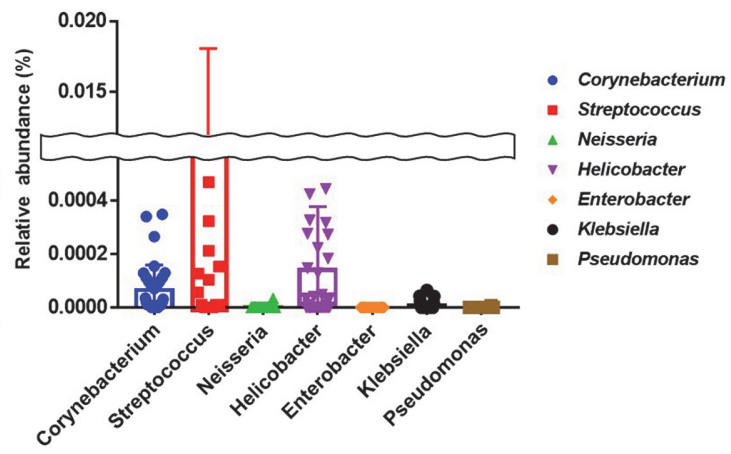

Fig. 6. Bacterial monitoring which can be related with foodborne illness $(A, B$, and $C)$ and potential pathogens $(D, E$, and $F)$ in feces samples. Monitoring for foodborne pathogens were classified 5 of genera and 2 of species in feces samples from (A) cattle, (B) chickens and (C) pigs. Monitoring of potential pathogens which can be opportunity pathogens were also conducted in feces samples from (D) cattle, (E) chickens and (F) pigs.

Campylobacter are asymptomatically carried in the animal's intestinal tract. They can be shed in feces in large populations and be transmitted by other vectors from feces to humans [17]. Foodborne pathogens may cause contamination of food not only by careless food management during the entire cooking process, but also by other food supply processes ranging from the production of livestock to the distribution process. It has been reported that agricultural products might be contaminated when livestock manure is used in soil as a raw material for compost $[3-5,18]$.

In this study, the presence or absence of foodborne pathogens was evaluated using a metagenomics approach. PCR-based method is usually used to detect and confirm the presence or absence of a toxin gene in targeted foodborne bacteria $[19,20]$. However, metagenomics sequencing techniques as culture-independent methods are currently widely used to identify and characterize microbiota. Therefore, we hypothesized that the metagenomics approach could be used to detect foodborne pathogens in samples such as foods, feces, and soils.

Campylobacteriosis caused mainly by genus Campylobacter is a zoonosis of increasing importance in industrialized countries. The most common routes of infection to humans are assumed to be consumption of contaminated raw or undercooked poultry and red meats and contaminated raw milk [21]. At present, more than $90 \%$ of Danish human Campylobacter cases are caused by C. jejuni, whereas approximately $5 \%$ of cases are diagnosed as C. coli [22]. National monitoring for antimicrobial resistance in Denmark has shown that the prevalence of $C$. jejuni is between 3 and $6 \%$ in finisher pigs over the past 5 years ( $1999 \sim 2003$ ) [23, 24]. However, other studies conducted in the United States, Canada, and Europe have shown that 30 to 50\% of Campylobacter isolates found in pigs and pork are C. jejuni [25-29]. Campylobacter was detected in cattle and pig feces in the present study, with pig feces having higher relative abundance of Campylobacter than cattle feces. Thus, we should be aware of the potential contamination of this pathogen in beef and pork products.

Moreover, Clostridium was detected in feces of three different species in our study. Clostridium perfringens is found in the environment. It can cause illness in both humans and animals. C. perfringens is found in soil, water from rivers and sewage, intestines, and feces of mammals including humans. Symptoms caused by C. perfringens include diarrhea and stomach cramps within 6 24 hours after infection [30]. C. perfringens causes foodborne illness through producing toxins of five types ( $\mathrm{A}, \mathrm{B}, \mathrm{C}, \mathrm{D}$, and $\mathrm{E})$. Type $\mathrm{A}$ is associated with foodborne pathogens in humans. It produces Clostridium perfringens alpha toxin (CPA) along with enterotoxin (CPE) with or without Clostridium perfringens beta 2 toxin (CPB2) [31,32]. It has been reported that $C$. perfringens is readily detected in foods such as pork, beef, chicken, and sheep [33-35]. In this study, the relative abundance of $C$. perfringens in chicken feces was the highest among three different livestock species. C. perfringens has been frequently detected in the intestinal tract and processed poultry meat. When intestinal contents of broiler chickens were analyzed for the presence of C. perfringens, approximately $75 \%$ to $95 \%$ of animals were found to be positive [36]. Furthermore, 
a previous report from Iran has shown that cpe gene of C. perfringens was founded in $27 \%$ and $32.76 \%$ intestinal contents of tested cattle and poultry, respectively [37].

Staphylococcus aureus is one of main public health concerns worldwide. It is a dangerous human pathogen that causes Staphylococcal food-borne disease (SFD) [38-40]. The virulence of $S$. aureus is mainly related to the production of a variety of protein toxins. The production of enterotoxins is associated with sepsis-related infections, food poisoning, pneumonia, and toxic shock syndrome [41]. It has been shown that staphylococcal enterotoxins (SEs) take an important role in the manifestation of a variety of human diseases [42, 43]. Chicken is the most common source of $S$. aureus in the world including South Korea. In this study, Staphylococcus was also detected in cattle, chicken, and pig samples at the genus level, with chicken feces having the highest relative abundance of those genera.

In addition, potential pathogens such as Helicobacter and Pseudomonsas were also identified in this study. The relative abundance of Helicobacter was the highest in pig feces and the relative abundance of Pseudomonsas was high in chicken and cattle fecal samples used in this study.

Helicobacter is a Gram-negative bacterium with a helical shape [44]. Helicobacter pylori is the most important pathogen that causes gastric diseases in humans. However, non-Helicobacter pylori helicobacter species could cause a disease in human as well as several animals [45]. Helicobacter species are commonly found in pig stomach [46].

Genus Pseudomonas contains 191 known species [47]. Certain Pseudomonas species including P. syringae, P. oryzihabitans, and P. aeruginosa can cause diseases in humans at hospital environments and animals [48-50]. Nosocomial infections of Pseudomonas can cause gastrointestinal infections and bacteremia [51]. Most Pseudomonas can produce polysaccharide which is associated with biofilm formation, resulting in resistance to phagocytosis by white blood cells. Most Pseudomonas are also naturally resistant to the majority of related betalactam antibiotics and penicillin [52].

Overall, we showed that microbiota of cattle, chicken, and pig feces were significantly different, and the distribution of foodborne pathogens in different livestock feces was also clearly different. Our results shall shed a new light on the food-borne risk assessments of livestock using metagenomics even with the limitation. Nevertheless, the accumulative and systematic analyses will help us to obtain a more reliable and general tendency in bacterial contamination in livestock feces and the further validation tests, such as isolation and identification of pathogens using selective medium or real-time PCR would be essential to present more reliable results from the metagenomics study. Even though identification of foodborne pathogens at genus level is limited, this study highlights the feasibility of using metagenomics approach to assess foodborne risk of livestock feces using metagenomics even with the limitation. However, the further studies using whole metagenome shotgun sequencing to detect toxin genes would warrant the identification of pathogenic traits in foodborne pathogens

\section{Acknowledgments}

The present study was supported by the research fund (19162MFDS037) from the Ministry of Food and Drug Safety, Republic of Korea.

\section{Conflict of Interest}

The authors have no financial conflicts of interest to declare.

\section{References}

1. Oliver SP, Jayarao BM, Almeida RA. 2005. Foodborne pathogens in milk and the dairy farm environment: food safety and public health implications. Foodborne Pathog. Dis. 2: 115-129.

2. Velusamy V, Arshak K, Korostynska O, Oliwa K, Adley C. 2010. An overview of foodborne pathogen detection: in the perspective of biosensors. Biotechnol. Adv. 28: 232-254.

3. R.Beuchat L. 1999. Listeria monocytogenes: incidence on vegetables Food Control 7: 6.

4. Heiman KE, Garalde VB, Gronostaj M, Jackson KA, Beam S, Joseph L, et al. 2016. Multistate outbreak of listeriosis caused by imported cheese and evidence of cross-contamination of other cheeses, USA, 2012. Epidemiol. Infect. 144: 2698-2708.

5. Fawcett AMSL. 2002. Tracking and traceability in the meat processing industry: a solution. Br. Food J. 104: 13.

6. Zhao X, Lin CW, Wang J, Oh DH. 2014. Advances in rapid detection methods for foodborne pathogens. J. Microbiol. Biotechnol. 24: 297-312.

7. Lundberg DS, Lebeis SL, Paredes SH, Yourstone S, Gehring J, Malfatti S, et al. 2012. Defining the core Arabidopsis thaliana root microbiome. Nature 488: 86-90.

8. Janda JM, Abbott SL. 2007. 16S rRNA gene sequencing for bacterial identification in the diagnostic laboratory: pluses, perils, and pitfalls. J. Clin. Microbiol. 45: 2761-2764.

9. Kim M, Lee KH, Yoon SW, Kim BS, Chun J, Yi H. 2013. Analytical tools and databases for metagenomics in the next-generation sequencing era. Genomics Inform. 11: 102-113.

10. Ku HJ, Lee JH. 2014. Development of a novel long-range 16S rRNA universal primer set for metagenomic analysis of gastrointestinal microbiota in newborn infants. J. Microbiol. Biotechnol. 24: 812-822.

11. Hanshew AS, Mason CJ, Raffa KF, Currie CR. 2013. Minimization of chloroplast contamination in 16S rRNA gene pyrosequencing of insect herbivore bacterial communities. J.Microbiol. Methods 95: 149-155.

12. Edgar RC, Haas BJ, Clemente JC, Quince C, Knight R. 2011. UCHIME improves sensitivity and speed of chimera detection. Bioinformatics 27: 2194-2200.

13. Caporaso JG, Kuczynski J, Stombaugh J, Bittinger K, Bushman FD, Costello EK, et al. 2010. QIIME allows analysis of highthroughput community sequencing data. Nat. Methods 7: 335-336.

14. Cole JR, Chai B, Farris RJ, Wang Q, Kulam SA, McGarrell DM, et al. 2005. The Ribosomal Database Project (RDP-II): sequences and tools for high-throughput rRNA analysis. Nucleic Acids Res. 33: D294-296. 
15. José A Navas-Molina JMP-S, Antonio González, Paul J McMurdie, Yoshiki Vázquez-Baeza, Zhenjiang Xu, Luke K Ursell, et al. 2013. Advancing our understanding of the human microbiome using QIIME. Methods Enzymol. 531: 371.

16. Parks DH, Beiko RG. 2010. Identifying biologically relevant differences between metagenomic communities. Bioinformatics 26: 715-721.

17. Doyle MP, Erickson MC. 2006. Reducing the carriage of foodborne pathogens in livestock and poultry. Poult. Sci. 85: 960-973.

18. Burnett SL, Beuchat LR. 2001. Human pathogens associated with raw produce and unpasteurized juices, and difficulties in decontamination. J. Ind. Microbiol. Biotechnol. 27: 104-110.

19. Rahn K, De Grandis SA, Clarke RC, McEwen SA, Galan JE, Ginocchio C, et al. 1992. Amplification of an invA gene sequence of Salmonella typhimurium by polymerase chain reaction as a specific method of detection of Salmonella. Mol. Cell. Probes 6: 271-279.

20. Miller RR, Montoya V, Gardy JL, Patrick DM, Tang P. 2013. Metagenomics for pathogen detection in public health. Genome Med. 5: 81.

21. Friedman CR, Neimann J, Wegener HC, Tauxe RV. 2000.

22. Anonymous. 2004. Annual Report on Zoonoses in Denmark 2003

23. Anonymous. 2000. Annual report on zoonoses in Denmark 1999.

24. Anonymous. 2002. Annual report on zoonoses in Denmark 2001.

25. Weber A, Lembke C, Schafer R. 1985. [Isolation of Campylobacter jejuni and Campylobacter coli in fecal samples of healthy slaughter swine depending on the season]. Zentralbl. Veterinarmed. B. 32: 40-45.

26. Finlay RC, Mann ED, Horning JL. 1986. Prevalence of Salmonella and Campylobacter contamination in manitoba Swine carcasses. Can. Vet. J. 27: 185-187.

27. Lammerding AM, Garcia MM, Mann ED, Robinson Y, Dorward WJ, Ruscott RB, et al. 1988. Prevalence of Salmonella and thermophilic Campylobacter in fresh pork, beef, veal and poultry in Canada. J. Food Prot. 51: 47-52.

28. Moore JE, Madden RH. 1998. Occurrence of thermophilic Campylobacter spp. in porcine liver in Northern Ireland. J. Food Prot. 61: 409-413

29. Harvey RB, Young CR, Ziprin RL, Hume ME, Genovese KJ, Anderson RC, et al. 1999. Prevalence of Campylobacter spp. isolated from the intestinal tract of pigs raised in an integrated swine production system. J. Am. Vet. Med. Assoc. 215: 1601-1604.

30. Brynestad S, Granum PE. 2002. Clostridium perfringens and foodborne infections. Int. J. Food Microbiol. 74: 195-202.

31. Suh J PO, Kang Y, Ahn JE, Jung JS, An YS, Park SH, et al. 2013. Risk assessment on nitrate and nitrite in vegetables available in Korean diet. J. Appl. Biol. Chem. 56: 205-211.

32. Mafart P, Couvert O, Gaillard S, Leguerinel I. 2002. On calculating sterility in thermal preservation methods: application of the Weibull frequency distribution model. Int. J. Food Microbiol. 72: 107-113.

33. Anonymous. 2018. Foodborne disease outbreak.

34. Hu WS, Kim H, Koo OK. 2018. Molecular genotyping, biofilm formation and antibiotic resistance of enterotoxigenic Clostridium perfringens isolated from meat supplied to school cafeterias in South Korea. Anaerobe 52: 115-121.

35. Jeong D, Kim DH, Kang IB, Chon JW, Kim H, Om AS, et al. 2017. Prevalence and toxin type of Clostridium perfringens in beef from four different types of meat markets in Seoul, Korea. Food Sci. Biotechnol. 26: 545-548.

36. Van Immerseel F, De Buck J, Pasmans F, Huyghebaert G, Haesebrouck F, Ducatelle R. 2004. Clostridium perfringens in poultry: an emerging threat for animal and public health. Avian Pathol. 33: 537-549.

37. Asghar Arshi SN, Hamidreza Kabiri, Arman Akbarpour, Rassoul Hashemzehi, Behnaz Mansouri, Ayse Kilic, et al. 2017. Incidence of Clostridium perfringens in intestinal contents of domestic livestock detected by PCR. Int. J. Anim. Res. 1: 1-6.

38. Balaban N, Rasooly A. 2000. Staphylococcal enterotoxins. Int. J. Food Microbiol. 61: 1-10.

39. Le Loir Y, Baron F, Gautier M. 2003. Staphylococcus aureus and food poisoning. Genet. Mol. Res. GMR 2: 63-76.

40. Hennekinne JA, De Buyser ML, Dragacci S. 2012. Staphylococcus aureus and its food poisoning toxins: characterization and outbreak investigation. FEMS Microbiol. Rev. 36: 815-836.

41. Lowy FD. 2003. Antimicrobial resistance: the example of Staphylococcus aureus. J. Clin. Invest. 111: 1265-1273.

42. Fraser JD, Proft T. 2008. The bacterial superantigen and superantigen-like proteins. Immunol. Revi. 225: 226-243.

43. Spaulding AR, Salgado-Pabon W, Kohler PL, Horswill AR, Leung DY, Schlievert PM. 2013. Staphylococcal and streptococcal superantigen exotoxins. Clin. Microbiol. Rev. 26: 422-447.

44. Baele M, Decostere A, Vandamme P, Ceelen L, Hellemans A, Mast J, et al. 2008. Isolation and characterization of Helicobacter suis sp. nov. from pig stomachs. Int. J. Syst. Evol. Microbiol. 58: 1350-1358.

45. Haesebrouck F, Pasmans F, Flahou B, Smet A, Vandamme P, Ducatelle R. 2011. Non-Helicobacter pylori Helicobacter species in the human gastric mucosa: a proposal to introduce the terms $H$. heilmannii sensu lato and sensu stricto. Helicobacter 16: 339-340.

46. Cantet F, Magras C, Marais A, Federighi M, Megraud F. 1999. Helicobacter species colonizing pig stomach: molecular characterization and determination of prevalence. Appl. Environ. Microbiol. 65: 4672-4676.

47. Lowy FD. 1998. Staphylococcus aureus infections. New Eng. J. Med. 339: 520-532.

48. Freney J, Hansen W, Etienne J, Vandenesch F, Fleurette J. 1988. Postoperative infant septicemia caused by Pseudomonas luteola (CDC group Ve-1) and Pseudomonas oryzihabitans (CDC group Ve-2). J. Clin. Microbiol. 26: 1241-1243.

49. Arnold DL, Preston GM. 2019. Pseudomonas syringae: enterprising epiphyte and stealthy parasite. Microbiology 165: 251-253.

50. Diggle SP, Whiteley M. 2020. Microbe Profile: Pseudomonas aeruginosa: opportunistic pathogen and lab rat. Microbiology 166: 30-33.

51. Van Delden C, Iglewski BH. 1998. Cell-to-cell signaling and Pseudomonas aeruginosa infections. Emerg. Infect. Dis. 4: 551-560.

52. Ryan KJ, Ray CG, Sherris JC. 2004. Sherris medical microbiology: an introduction to infectious diseases, pp. 997. $4^{\text {th }}$ Ed. McGrawHill, New York, USA 